\title{
DNA damage and cell death assessment in patients with severe multiple trauma using comet assay
}

\author{
Aliy K. Zhanataev ${ }^{1 *}$, Victor V. Moroz², Andrey D. Durnev, Maria Yu. Muravyeva², \\ Vasiliy I. Reshetnyak ${ }^{2}$

\footnotetext{
${ }^{1}$ Science Research Institute of Pharmacology, Russian Academy of Medical Sciences, Moscow, Russia; *Corresponding Author: azhanataev@yandex.ru

${ }^{2}$ Science Research Institute of General Reanimatology, Russian Academy of Medical Sciences, Moscow, Russia; v reshetnyak@yahoo.com
}

Received 9 December 2009; revised 20 February 2010; accepted 23 February 2010.

\begin{abstract}
Purpose: To determine the DNA strand breaks, oxidative DNA damage and cell death in blood and plasma total antioxidant status (TAOS) in 22 patients with severe multiple trauma. Materials and methods: The DNA comet assay was used to measure DNA strand breakage, 8-oxoguanine levels and apoptotic and necrotic nuclei in after admission (day 0) and on days 3, 5, 7 and 15. TAOS was determined by colorimetric method. Results: Trauma patients had high DNA damage at admission $(p<0.01)$, that further increased with maximum value on day $5(p<0.001)$. On day 15 the degree of DNA damage remained significantly elevated $(p<0.01)$. No significant difference in the 8-oxoguanine levels at all days examined was found. Patients had a high percentage of apoptotic and necrotic comets at admission, with maximum values on days 3 and 5. A significantly lower TAOS was observed in patients on admission and days $3,5,7$ and 15 (p $<0.001$ in all cases). A decreasing of TAOS on days 7 and 15 compared to that on admission ( $p$ $<0.05$ ) was observed. Conclusions: Blood cells from severe trauma patients' display increased DNA damage associated with apoptosis and necrosis. Reduced plasma TAOS and a tendency to increase of 8-oxoguanine in DNA was observed.
\end{abstract}

Keywords: Apoptosis; Necrosis; Multiple Trauma; DNA-Comet Assay; 8-Oxoguanine;

Total Antioxidant Status

\section{INTRODUCTION}

Severe multiple trauma remains as one of the major problems of contemporary medicine and society throughout the world. Advances in critical care of the trauma patient have resulted in improved outcome, but despite these efforts, up to half of the patients with traumatic injury die or are left with severe disability [1-3].

Multiple organ failure (MOF) is the major complication after sever multiple trauma [4-7]. Experimental evidences suggest that MOF in the event of trauma might be relates to stress-induced cell death by apoptosis, but its exact mechanisms is not fully understood [8-12]. DNA damage including oxidative DNA bases modification is one of the intrinsic signals initiating apoptosis [13]. At present, data about DNA damage in critical illness are lacking. The Single Cell Gel Electrophoresis assay (SCGE), or comet assay are sensitive method for the evaluation of DNA damage from individual cells based on the migration of denatured DNA through an electrophoretic field [14]. The aim of this pilot study was an assessment DNA strand breakage, 8-oxoguanine levels and cellular death of white blood cells and their possible connection with total antioxidant status in patients with severe multiple trauma.

\section{MATERIALS AND METHODS}

The study covered 22 patients who were admitted with severe multiple traumas (SMT) (Table 1). The study protocol was approved by ethics committee and informed consent was obtained from all patients or their relatives. Inclusion criteria were age older than 18 years, admission to the trauma intensive care unit and an Acute Physiology and Chronic Health Evaluation (APACHE II) score higher than 12 . Patients with traumatic brain injury were excluded. The age ranged from 21 to 68 years. Body weight ranged from 50 to $95 \mathrm{~kg}$. Clinical characteristics of the patients with severe multiple trauma on admission is presented to Table 2. The length of stay in the unit ranged from 3 to 32 days, with a mean of $10.5 \pm$ 
5.1 days. The patient's condition on admission was an APACHE II of $19.1 \pm 5.4$, blood loss $22-45 \mathrm{ml} / \mathrm{kg}$. Blood loss was calculated using indirect method (vital signs, serial hematocrits measurement) and by direct intraoperative estimation of blood loss. Optimization of hemodynamic (systolic blood pressure $>90 \mathrm{~mm} \mathrm{Hg}$ ) and oxygenation (arterial oxygen saturation $>90 \%$ ) was reached during the first hours after the admission in the intensive care unit. Single organ failure (SOF) occurred in 12 patients $(54.5 \%)$ and multiple organ failure occurred in 6 patients $(27.3 \%)$. All SOF was caused by respiratory failure. Respiratory failure occurred first in the majority of patients with multiple organ failure, followed usually by cardiovascular insufficiency. The mortality level was $23 \%$ (5 patients). Blood was collected after admission (day 0$)$ within 4 hours $\left(\mathrm{n}_{0}=22\right)$, and on days $3\left(n_{3}=22\right), 5\left(n_{5}=13\right), 7\left(n_{7}=11\right)$ and $15\left(n_{15}=5\right)$ of admission. Blood samples $(2 \mathrm{ml})$ were taken into EDTA tubes, mixed (1:1) with RPMI-1640 medium containing 20\% DMSO as cryoprotectant and immediately frozen to $-20^{\circ} \mathrm{C}$. Samples stored until analysis no more 20 days avoiding repeated freeze-thaw cycles. Twelve subjects without acute or chronic disease were used as control group (Table 1). Blood samples from controls handled and stored using identical procedures.

\subsection{Single Cell Gel Electrophoresis Assay}

DNA damage was evaluated using alkaline comet assay [15]. Briefly, after quick (within 1-2 min) thawing in a water-bath at $37^{\circ} \mathrm{C}, 50 \mu \mathrm{l}$ of whole blood samples were mixed with $500 \mu \mathrm{l}$ of $1 \%$ melted agarose (low melting point) and layered $(70 \mu \mathrm{l})$ onto a microscopy slide. The slides with the agarose-embedded cells were subjected to a lysis step $(1 \mathrm{~h}$ at $40 \mathrm{C}$ in $1 \%$ N-lauroylsarcosine, $2.5 \mathrm{M}$ $\mathrm{NaCl}, 100 \mathrm{mM} \mathrm{Na}{ }_{2}$ EDTA, $1 \%$ Triton X-100, 10\% DMSO, $\mathrm{pH}$ 10.0). After the lysis step slides were placed in an ice-cold electrophoresis chamber containing alkaline electrophoresis solution $(300 \mathrm{mM} \mathrm{NaOH}, 1 \mathrm{mM}$ $\mathrm{Na}_{2}$ EDTA, $\mathrm{pH}>13.0$ ) for 20 min to allow DNA unwinding. The electrophoresis was subsequently conducted for $20 \mathrm{~min}$ at $1 \mathrm{~V} / \mathrm{cm}$ and $\sim 300 \mathrm{~mA}$. At the end of the electrophoresis the slides were fixed in $70 \%$ ethanol, air-dried and stored in the dark at room temperature until scored. Just prior to scoring, slides were stained with SYBR Green I (1:10000 in TE-buffer, pH 7.4) for 20 min. Microscopical analysis was carried out at $\times 200$ magnification using MIKMED-2 microscope (LOMO, Russia) provided with epifluorescence and equipped with a FITC filters. Images of 100 randomly selected comets were captured using CCD camera (VEC-335, EVS, Russia) and analyzed with CASP 1.1.2 image analyzer software [16] to evaluate \%DNA in tail (the percentage of total fluorescence migrated in the tail for each nucleus) used as a measure of DNA damage. In parallel analysis levels of apoptotic and necrotic comets was estimated. Comet assay in the standard alkaline version
Table 1. Demographic data of the investigated groups.

\begin{tabular}{lcc}
\hline \multicolumn{1}{c}{ Parameter } & $\begin{array}{c}\text { Trauma patients } \\
(\mathrm{n}=22)\end{array}$ & $\begin{array}{c}\text { Healthy subjects } \\
(\mathrm{n}=12)\end{array}$ \\
\hline $\mathrm{M} / \mathrm{F}, \mathrm{n}(\%)$ & $14(63,6 \%) / 8(36,4 \%)$ & $5(41,7 \%) / 7(58,3 \%)$ \\
Age, years & $41.9 \pm 13.3$ & $37.9 \pm 9.6$ \\
Weight, kg & $75.7 \pm 6.4$ & $67.8 \pm 7.5$ \\
Motor vehicle accident & $18(81,8 \%)$ & - \\
Fall & $2(9,1 \%)$ & - \\
Other mechanism of & $2(9,1 \%)$ & - \\
injury & $16(72,7 \%)$ & - \\
Blunt trauma & $6(27,3 \%)$ & - \\
Penetrating trauma & & \\
\hline
\end{tabular}

has a fragment size resolution of $10-100 \mathrm{~kb}$ and early to middle chromatin fragmentation during apoptosis can be monitored by the technique [17]. Thus, DNA-comets of apoptotic cells on slides clearly differ from damaged or undamaged cells by forming comet- like structures with spread tail and small almost invisible heads (Figure 1(b)) $[14,17]$. Necrotic cells displayed a characteristic view with large nuclear remnants which reflected random DNA degradation (Figure 1(c)).

\subsection{Detection of 8-Oxoguanine and TAOS}

Human 8-oxoguanine DNA Glycosylase (hOGG1) FLARE Assay Kit (R\&D Systems, Minneapolis, USA) was used to evaluate oxidative DNA damage in blood cells. This test uses the hOGG1 enzyme, a glycosylase that recognizes and specifically cuts the oxidized bases principally 8-oxoguanine (8-oxoG) from DNA, producing apurinic sites converted in breaks, which can be detected by comet assay. The comet assay was carried out as described above, with the exception that after lysis of cells the slides were washed three times for 15 min with FLARE $^{\mathrm{TM}}$ buffer. After this time samples were incubated with $100 \mu$ of hOGG1 enzyme (1:400 in REC dilution buffer) or with $100 \mu \mathrm{l}$ of REC dilution buffer only. Slides incubated in humidity chamber at $37^{\circ} \mathrm{C}$ for $1 \mathrm{~h}$. DNA unwinding, electrophoresis, microscopic analysis and comet scoring were then completed as described above. For each blood sample the mean values of the $\%$ DNA in tail for 100 comets from hOGG1-treated cells (\%DNAenz) and hOGG1-untreated cells (\%DNAbuf) was calculated. \%DNAenz/\%DNAbuf ratio (in a.u.) was used as measure of 8-oxoG content in DNA of blood cells.

Among the methodologies used to evaluate TAOS, the most widely used colorimetric method are 2,2 '-azinobis3 -ethylbenzothiazoline-6-sulfonic acid radical cation $($ ABTS + ) based methods. TAOS of plasma samples was assayed by using a commercially available kit (Randox Laboratories Ltd., Crumlin, UK) on an automatic Cobas 
Mira Plus Chemistry analyzer (Roche Diagnostics Ltd., USA). The results were expressed as mmol/l Trolox equivalent.

\subsection{Statistical Analysis}

Data are presented as mean $\pm \mathrm{SD}$. Statistical comparisons between controls and patients were performed by Student's t-test for independent samples. A Wilcoxon matched pairs signed rank test was used to compare paired samples. Correlations were evaluated using Spearman's rank correlation test. A value of $p<0.05$ was considered as statistically significant.

\section{RESULTS}

Typical DNA-comet images of blood cells taken from healthy individuals and patients with SMT are presented in Figure 1. Comparison of DNA damage in blood cells

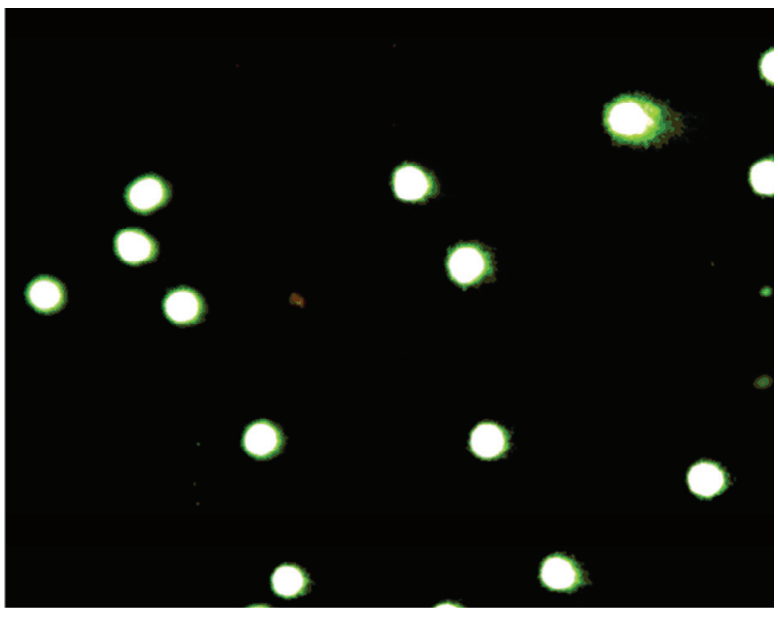

(a)

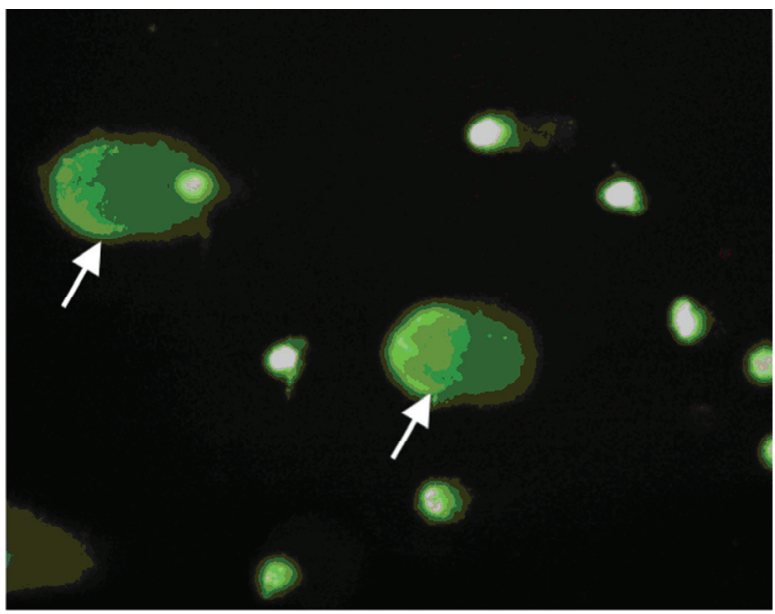

(c) from patients with SMT and healthy controls is presented in Figure 2(a). Tail DNA percentage in blood cells from healthy controls ranged from 2.4 to 10.4 with mean value $6.1 \pm 2.2 \%$. A significantly higher level of DNA damage was seen in patients on the day of admission $(10.6 \pm 5.9 \% ; \mathrm{p}<0.01)$. The further increase in DNA damage levels was observed up to day 7 with maximum value on day $5(14.2 \pm 4.2 \%$ DNA in tail; $\mathrm{p}<$ $0.001)$. On day 15 the degree of DNA damage returned to the admission value, but remained significantly elevated in comparison with healthy controls $(6.1 \pm 2.2 \%$ vs. $10.3 \pm 2.4 ; \mathrm{p}<0.01)$.

There was no significant difference in the levels of 8-oxoG between controls and trauma patients at all days examined (Figure 2(b)). A marked increase in 8-oxoG levels was observed on day 7 (up to $2.1 \pm 0.6$ a.u.). Pair wise comparison indicated that this increase was not significant.

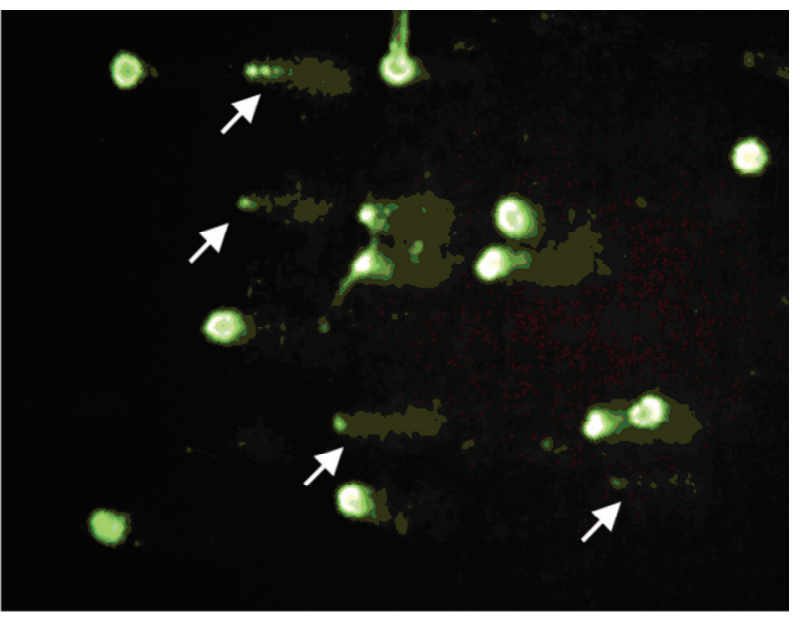

(b)

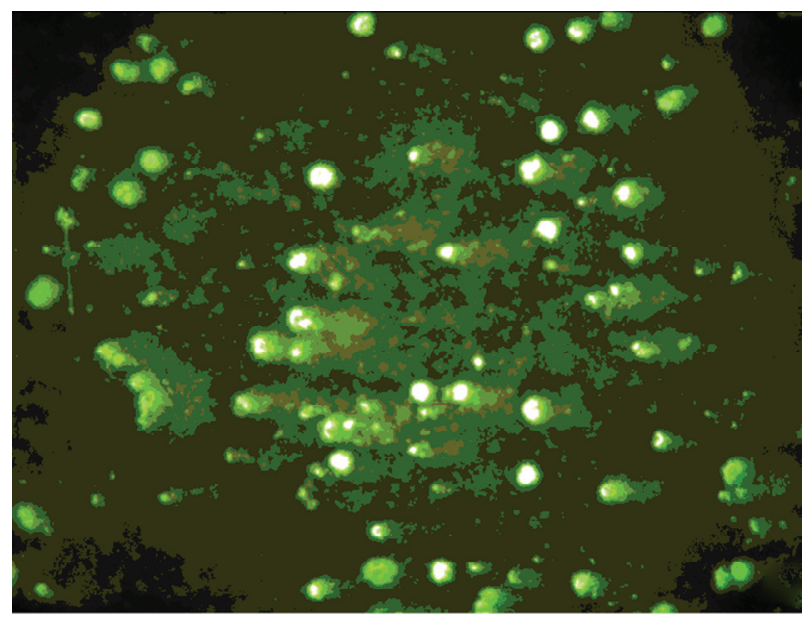

(d)

Figure 1. Typical DNA-comet images of blood cells taken from a control subject (a) and patients with severe multiple trauma (b,c,d). Arrows indicates DNA-comets of apoptotic (b) and necrotic (c) nuclei. Image from blood sample with high-fragmented DNA are showed (d). Magnification $\times 200$. 
Healthy individuals showed low level of spontaneously apoptosis of blood cells $(0.9 \pm 0.3 \%$ of apoptotic comets; Figure 2(c)). In contrast, percentage of apoptotic comets was found elevated in patients at day of admission $(2.7 \pm 2.6 \%$, range from 0.4 to $10.3 ; \mathrm{p}<0.01)$. By day 3 mean apoptosis rate increased up to $6.9 \pm 7.7 \%$ $(\mathrm{p}<0.001)$ with widely varied values from 0.7 to $32 \%$. From day 5 apoptotic comets score gradually decreased to value $2.3 \pm 1.1 \%$ on day 15 . No necrotic DNA-comets were found in slides from controls' blood samples. The mean percentage of necrotic comets in patients was $4.9 \pm$ $5.9 \%$ at day 0 (Figure 2(d)). Further increasing to maximum value of $9.3 \pm 7.1 \%$ on day 5 was observed, but from day 7 to day 15 necrotic DNA-comets reduced to $5.0 \pm 3.6 \%$. No any statistical differences at pairwise comparison were found due to high interindividual diversity and insufficient number of patients.

A statistically significantly lower TAOS values (Figure 2(e)) was observed in patients on admission $(0.84 \pm$ $0.22 \mathrm{mmol} / \mathrm{l})$ and days $3,5,7$ and $15(0.74 \pm 0.23 ; 0.76$ $\pm 0.26 ; 0.68 \pm 0.25$ and $0.59 \pm 0.16 \mathrm{mmol} / 1$, respectively) as compared to healthy subjects $(1.34 \pm 0.25 \mathrm{mmol} / \mathrm{l} ; \mathrm{p}<$ 0.001 in all cases). A statistically significant decreasing of TAOS on days 7 and 15 was also observed compared to that on admission $(\mathrm{p}<0.05)$.

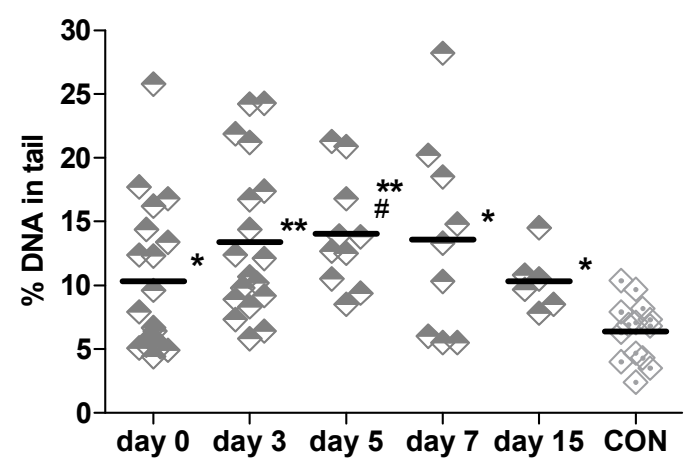

(a)

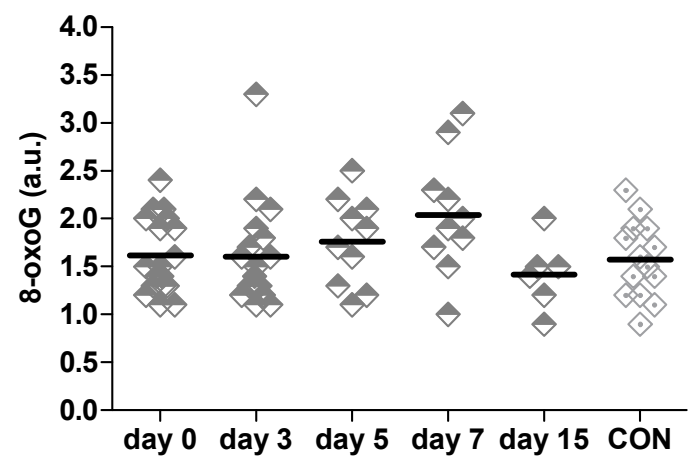

(b)

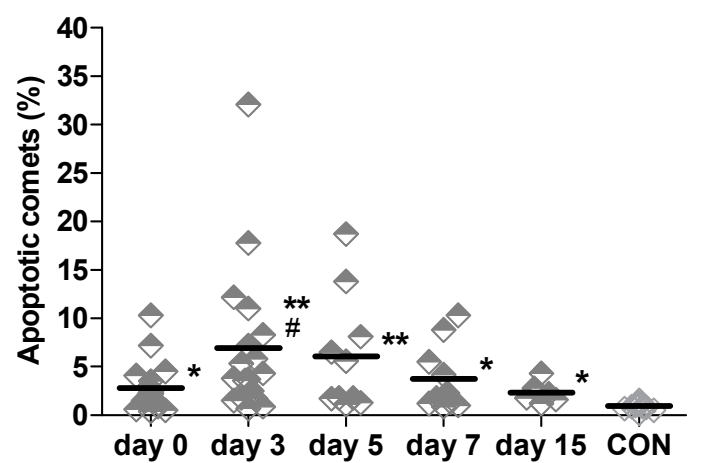

(c)

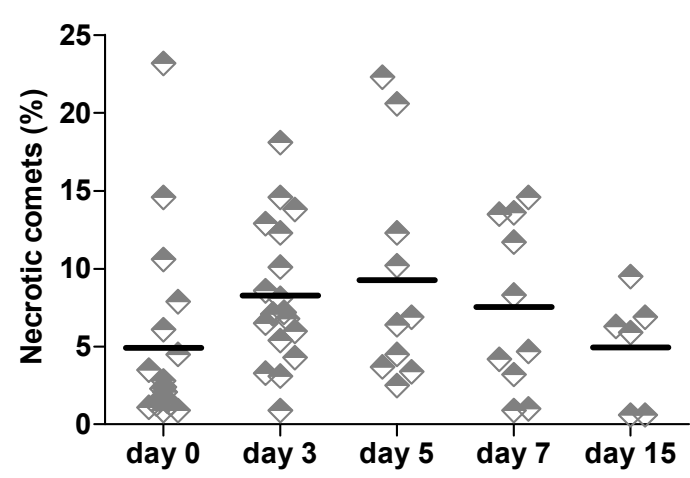

(d)

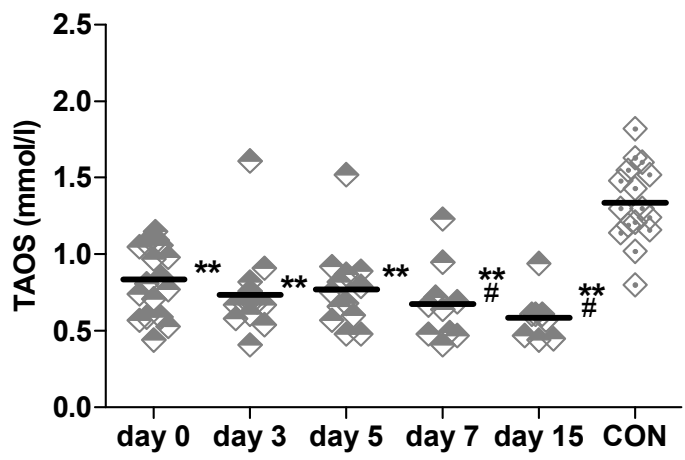

(e)

$*_{-}-p<0.01$ as compared with controls; **- $p<0.001$ as compared with controls; \# $-p<0.05$ as compared with value at day 0.

Figure 2. DNA damage (a), 8-oxoG levels (b), apoptotic (c) and necrotic (d) comets percentage in blood cells and plasma total antioxidant status (e) in healthy controls (CON) and patients with severe multiple trauma at different days of analysis.

Correlation analysis demonstrated that there is a significant direct relationship between \%DNA in tail and percentage of necrotic comets on day $0(r=0.67 ; \mathrm{p}<$ $0.001)$ and day $3(r=0.54 ; \mathrm{p}<0.01)$ and between $\%$ DNA in tail and percentage of apoptotic comets on day $7(r=0.69 ; p<0.001)$. A significant negative correlation 
between \%DNA in tail and 8-oxoG levels on day $0(r=$ $-0.71 ; \mathrm{p}<0.001)$, day $3(r=-0.62 ; \mathrm{p}<0.001)$ and day 5 $(r=-0.74 ; \mathrm{p}<0.001)$ was found. No any correlations between TAOS and DNA damage, 8-oxoG, apoptotic or necrotic comets were found

\section{DISCUSSION}

The DNA comet assay is sensitive method for the evaluation of DNA damage from individual cells based on the migration of denatured DNA through an electrophoretic field [14]. Under alkaline conditions, comet assay detects single and double strand breaks, alkalilabile and abasic sites, and DNA cross-links. By adding modifying enzymes can be investigated specific types of DNA damage, such as oxidative damage of DNA bases [14]. Also, comet assay enable the discrimination of apoptotic and necrotic nuclei on the basis of their characteristic signature of DNA fragmentation patterns [14, 17]. Our data represent the first examination of the DNA damage in blood cells of patients with severe multiple trauma. A significantly higher level of DNA strand breaks, apoptotic and necrotic nuclei in blood cells of patients versus controls, beginning from day of admission were found. The analysis of the data allows assuming that the main processes connected with DNA damage and cell death at trauma occur from day 3 to day 5 . In our trial trauma has served as a model for investigation because the interval between triggering event and development of organ failure can be assessed precisely and primary organ injury can be easily separated from remote organ dysfunctions.

Moderate hypoxia and hypoperfusion (Table 2), organ and soft tissue injuries, fractures, as well as ische$\mathrm{mia} /$ reperfusion probably induced disbalance in pro- and antioxidative system and as result oxidative stress in patients. The low level of TAOS in patients beginning

Table 2. Clinical characteristics of the patients with severe multiple trauma on admission.

\begin{tabular}{lc}
\multicolumn{1}{c}{ Parameter } & Value \\
\hline $\mathrm{PaO}_{2} / \mathrm{FiO}_{2}$ & $267.54 \pm 44.32$ \\
Glasgo coma score & $13.5 \pm 1.3$ \\
Mean arterial pressure, $\mathrm{mm} \mathrm{Hg}$ & $64.1 \pm 23.2$ \\
Crystalloid infusion in 4 hours, $\mathrm{ml}$ & $4826 \pm 259$ \\
Lactate, $\mathrm{mmol} / \mathrm{l}$ & $3.7 \pm 1.45$ \\
Creatinin, $\mathrm{mg} / \mathrm{dl}$ & $0.82 \pm 0.48$ \\
Bilirubin, $\mathrm{mg} / \mathrm{dl}$ & $0.96 \pm 0.32$ \\
Initial hemoglobin level, $\mathrm{g} / \mathrm{l}$ & $112.3 \pm 15.4$ \\
Platelets, $\times 10^{3} / \mathrm{ml}$ & $210.7 \pm 38.2$ \\
\hline
\end{tabular}

from day of admission with a progressive $42 \%$ reduction during the next two weeks was observed. Several studies indicate that oxidative stress occurs in critical illness $[9,19,20]$. The presence of $8-0 x o G$ in DNA is considered a marker of oxidative DNA damage. Using HPLC with electrochemical detection elevated oxidative DNA damage in trauma patients as measured by estimating 8oxodG:dG ratios in DNA of blood cells was shown [20]. However, in this study using comet FLARE assay no differences in 8-oxoG levels were observed, despite a markedly reduced TAOS. On the one hand it might be connected with intensive DNA repair. Alkaline comet assay detects strand breaks, alkali-labile sites, as well as abasic sites-excision repair sites missing either a pyrimidine or purine nucleotide, and the observed high level of DNA breaks might reflect the high repair activity. The presence of an inverse relationship between DNA breaks and 8-oxoG levels observed on days 0,3 and 5 may support this hypothesis. On the other hand elimination of cells with oxidatively damaged DNA through apoptosis/necrosis can lead to an underestimation of 8-oxoG. So, necrotic and apoptotic comets have been excluded from 8-oxoG analysis due to methodological features. Data will allow assuming that evaluation of repair product of 8-oxoG-8-hydroxy-2-deoxyguanosine (8-oxodG) in plasma and/or 8-oxoG content in circulating plasma cell-free DNA in future may be a more useful approach for estimating oxidative DNA damage in critical ill patients. Also we found that there were no significant correlations between DNA damage and TAOS. These results indicate that DNA damage at trauma may be caused not only by oxidative stress but also by other pathways.

In eight patients for days 3 and/or 5 the massive DNA fragmentation revealed on slides as a diffuse distributed high-fragmented DNA was observed (Figure 1(d)), complicating the analysis and a scoring of DNA comets. It is not clear, whether it is a DNA of necrotic or apoptotic cells. Pachl et al. showed that in critically ill patients both apoptotic and necrotic DNA contributed to total plasma DNA and its increase predicted future development of multiple organ failure and death [21]. The question remains open with regard to whether is this high-fragmented DNA a consequence apoptosis/necrosis of blood cells or this DNA eliminated in blood from damaged tissues and/or organs due to intensive cell death. Apoptosis in thymus, liver, lung, intestine and spleen after major trauma combined with shock has been demonstrated in animal experiments [22]. Non-lethal mechanical trauma causes significant TNF-alpha production that in turn stimulates myocardial apoptosis via oxidative/nitrative stress [23]. In patients with major trauma, early apoptosis was detected in lymphoid tissues [12]. It is necessary to notice that the exception of the analysis of the samples with high-fragmented DNA can explain absence of statistically significant differences at 
the analysis, despite obvious tendencies.

Continuing studies with a larger number of patients are required. Our results underscore the need to use the comet assay in two variants, the alkali and the neutral assays that allows a more complete study of DNA damage. Also, parallel analysis of plasma/serum DNA and DNA repair rate of oxidative DNA damage may help evaluate interrelations of different factors and its role in cellular death mechanisms and development MOF.

In conclusion, blood cells from severe trauma patients' display increased DNA damage associated with apoptosis and necrosis. Reduced plasma TAOS and a tendency to increase of 8-oxoguanine in DNA was observed.

\section{REFERENCES}

[1] Holtslag, H.R., van Beeck, E.F., Lindeman, E., et al. (2007) Determinants of long-term functional consequences after major trauma. Journal of Trauma, 62(4), 919-927.

[2] Soberg, H.L., Bautz-Holter, E., Roise, O., et al. (2007) Long-term multidimensional functional consequences of severe multiple injuries two years after trauma: A prospective longitudinal cohort study. Journal of Trauma, 62(2), 461-470.

[3] Stalp, M., Koch, C., Ruchholtz, S., et al. (2002) Standardized outcome evaluation after blunt multiple injuries by scoring systems: A clinical follow-up investigation 2 years after injury. Journal of Trauma, 52(6), 1160-1168.

[4] Nast-Kolb, D., Aufmkolk, M., Rucholtz, S., et al. (2001) Multiple organ failure still a major cause of morbidity but not mortality in blunt multiple trauma. Journal of Trauma, 51(5), 835-842.

[5] Durham, R.M., Moran, J.J., Mazuski, J.E., et al. (2003) Multiple organ failure in trauma patients. Journal of Trauma, 55(4), 608-616.

[6] Antonelli, M. and Caricato, A. (2007) Post-injury multiple organ failure and late outcome. Is it just an association? Critical Care, 11(5), 166.

[7] Walsh, C.R. (2005) Multiple organ dysfunction syndrome after multiple trauma. Orthopedic Nursing, 25(5), 324-333.

[8] Keel, M. and Trentz, O. (2005) Pathophysiology of polytrauma. Injury, 36(6), 691-709.

[9] Goodyear-Brunch, C. and Pierce, J.D. (2002) Oxidative stress in critically ill patients. American Journal of Critical Care, 11, 543-553.

[10] Cobb, P.J., Buchman, T.G., Karl, I.E., et al. (2000) Mo- lecular biology of multiple organ dysfunction syndrome: Injury, adaptation and apoptosis. Surgical Infection (Larchmt), 1(3), 207-215.

[11] Papathanassoglou, E.D., Moynihan, J.A. and Ackerman, M.H. (2000) Does programmed cell death (apoptosis) play a role in the development of multiple organ dysfunction in critically ill patients? A review and a theoretical framework. Critical Care Medicine, 28(2), 537549.

[12] Hotchkiss, R.S., Schmieg, R.E., Swanson, P.E., et al. (2000) Rapid onset of intestinal epithelial and lymphocyte apoptotic cell death in patients with trauma and shock. Critical Care Medicine, 28(9), 3207-3217.

[13] Norbury, C.J. and Zhivotovsky, B. (2004) DNA damage-induced apoptosis. Oncogene, 23, 2797-2808.

[14] Collins, A.R. (2004) The comet assay for DNA damage and repair: principles, applications, and limitations. Molecular Biotechnology, 26(3), 249-261.

[15] Tice, R.R., Agurell, E., Anderson, D., et al. (2000) Single cell gel/comet assay: Guidelines for in vitro and in vivo genetic toxicology testing. Environmental and Molecular Mutagenesis, 35(3), 206-221.

[16] Konca, K., Lankoff, A. and Banasik, A. (2003) A crossplatform public domain PC image-analysis program for the comet assay. Mutation Research, 534(1-2), 15-20.

[17] Morley, N., Rapp, A., Dittmar, H., et al. (2006) UVAinduced apoptosis studied by the new apo/necro-Cometassay which distinguishes viable, apoptotic and necrotic cells. Mutagenesis, 21(2), 105-114.

[18] Barzilai, A. and Yamamoto, K. (2004) DNA damage responses to oxidative stress. DNA Repair (Amst), 3(8-9), 1109-1115.

[19] Valko, M., Leibfritz, D., Moncol, J., et al. (2007) Free radicals and antioxidants in normal physiological functions and human disease. International Journal of Biochemistry \& Cell Biology, 39(1), 44-84.

[20] Oldham, K.M., Wise, S.R., Chen, L., et al. (2002) A longitudinal evaluation of oxidative stress in trauma patients. Journal of Parenteral and Enteral Nutrition, 26(3), 189197.

[21] Pachl, J., Duska, F., Waldauf, P., et al. (2005) Apoptosis as an early event in the development of multiple organ failure? Physiological Research, 54, 697-699.

[22] Guan, J., Jin, D.D., Jin, L.J., et al. (2002) Apoptosis in organs of rats in early stage after polytrauma combined with shock. Journal of Trauma, 52(1), 104-111.

[23] Li, S., Tao, L., Jiao, X., et al. (2007) TNFalpha-initiated oxidative/nitrative stress mediates cardiomyocyte apoptosis in traumatic animals. Apoptosis, 12(10), 1795-1802. 